\title{
Influencia de la jornada de trabajo en la salud del personal de enfermería
}

\section{Influence of shifts of work in the health of the personnel of nurses \\ Influência de turnos de trabalho na saúde do pessoal de enfermeiros}

Ninfa del Carmen Vega Monsalve

Magister en Gestión del Talento Humano. Corporación Universitaria Minuto de Dios-UNIMINUTO.

Escuela de Gestión Estratégica-EGE. Medellín Antioquia, Colombia.

Cómo citar este artículo en edición digital: Vega-Monsalve, N.D.C. (2018). Influencia de la jornada de trabajo en la salud del personal de enfermería. Cultura de los Cuidados (Edición digital), 22(50).

Recuperado de http://dx.doi.org/10.14198/cuid.2018.50.13

Correspondencia: Remitirse al correo electrónico.

Correo electrónico: ninfavegamonsalve@gmail.com

Recibido: 15/06/2017; Aceptado: 21/10/2017

Financiamiento: Investigación cofinanciada con recusos de la II convocatoria para el desarrollo y fortalecimiento de los Semilleros de Investigación de UNIMINUTO, 2015. Através del proyeto no. 520-1N-1-15-011

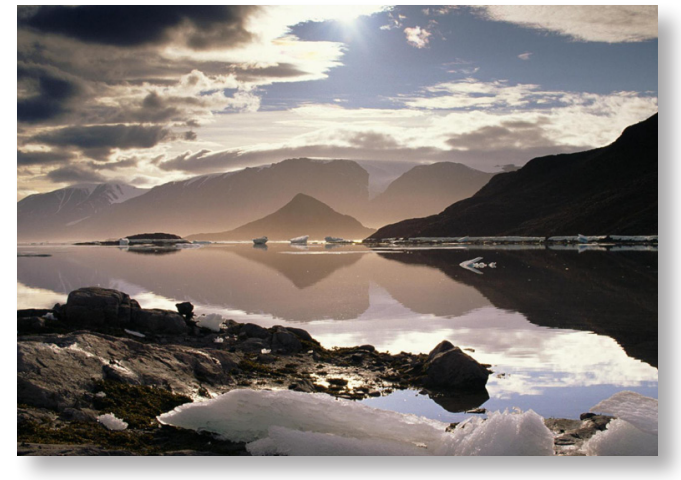

ABSTRACT

Objective: To understand the influence of work conditions in the health of Colombian nursing personnel, especially regarding the work shifts in Antioquia.

Methods: We performed a cross-sectional study on a sample of 365 nurses, men and women between 18 and 58 years old, with more than one year of experience in the profession, who performed night shifts in the last quarter and who work for public or private institutions of 39 municipalities of Antioquia's health department. We found an indicator of 5\% error and $98 \%$ confidence. Performing self-directed questionnaire scales and 21 semi-structured interviews. Descriptive statistic calculations were performed and statistical chi-square test was applied.

Results: We found that working conditions especially related to work shifts have a high influence on the health status of Colombian nurses in the territory of Antioquia, in comparison with other countries; working conditions are precarious, especially in the amount of working hours.

Conclusions: We conclude that working conditions detract the health conditions of nurses, so recommendations on how to reduce this influence are necessary.

Keywords: Occupational health, Nurses, Working conditions, Labor shifts.

\section{RESUMEN}

Objetivo: Comprender la influencia de las condiciones de trabajo referidas a jornadas laborales en la salud del personal de enfermería de Colombia, territorio Antioqueño.

Métodos: Este estudio aprovechó las bondades de los tipos de investigación cualitativa y cuantitativa. Luego de los cálculos estadísticos y análisis de los datos numéricos, se procedió a darle profundidad a los resultados, por medio de la comprensión de la incidencia de las 
condiciones de trabajo en la salud y vida del personal de enfermería, de allí la decisión de triangular métodos desde los instrumentos utilizados. Se realizaron 365 cuestionarios y 21 entrevistas. Los participantes fueron hombres y mujeres entre los 18 y 58 años de edad, con más de un año de experiencia en la profesión y que laboraban para instituciones de salud de 39 municipios de Antioquia.

Resultados: Las condiciones de trabajo especialmente referidas a jornadas laborales tienen una alta influencia en las condiciones de salud del personal de enfermería de Colombia del territorio Antioqueño, comparativamente con otros países las condiciones de trabajo son precarias, sobre todo en cuanto a duración de la jornada laboral.

Conclusiones: Se concluye que las condiciones de trabajo desmejoran las condiciones de salud del personal de enfermería.

Palabras clave: Salud laboral, Personal de enfermería, Condiciones de trabajo, Jornada laboral.

\section{RESUMO}

Objectivo: Para entender a influência das condições de trabalho na saúde do pessoal de enfermagem colombianas, principalmente em relação aos turnos de trabalho em Antioquia.

Métodos: Foi realizado um estudo transversal com uma amostra de 365 enfermeiros, homens e mulheres entre 18 e 58 anos, com mais de um ano de experiência na profissão, que realizou turnos noturnos no último trimestre e que trabalham para o público ou instituições privadas de 39 municípios do departamento de saúde de Antioquia. Encontramos um indicador de erro de 5\% e $98 \%$ de confiança. Realizando escalas questionário de auto-dirigida e 21 entrevistas semi-estruturadas. cálculos estatísticos descritivos foram re- alizados e foi aplicado o teste do qui-quadrado estatística.

Resultados: Verificou-se que as condições de trabalho, especialmente relacionadas com turnos de trabalho têm uma grande influência sobre o estado de saúde dos enfermeiros colombianos no território de Antioquia, em comparação com outros países, as condições de trabalho são precárias, especialmente na quantidade de horas de trabalho.

Conclusões: Conclui-se que as condições de trabalho prejudicar as condições de saúde das enfermeiras, de modo recomendações sobre como reduzir essa influência são necessárias.

Palavras-chave: Saúde do trabalhador, enfermeiros, Condições de trabalho, turnos de trabalho.

\section{INTRODUCCIÓN}

Distintos estudios (Romero-Ballén y Mesa-Melgarejo, 2010; Costa, 2010; Manenschijn, et al., 2011; Ramírez, Paravic y Valenzuela, 2013 y ASNEC, 2014) han indicado que las condiciones laborales del personal de enfermería inciden en el deterioro de la vida personal, social y familiar, actividad laboral y cumplimiento misional de la profesión. Estas condiciones pueden ser de dos tipos: intralaborales y extralaborales (Da Silva, 2006) y de acuerdo a las estrategias de afrontamiento personal con las que cuente el enfermero o las condiciones del medio, pueden ser mayormente favorecedoras u obstaculizadoras del logro de los objetivos organizacionales y el desarrollo personal del trabajador.

Aunque se conoce de antemano que el trabajo hospitalario es intenso y que la carga física y mental sumada a extensos horarios provoca múltiples efectos en los empleados (Velandia, 2011), algunas de las condiciones 
de trabajo de los enfermeros en Colombia están por debajo de la realidad de otros países de la región como Argentina (Ley 24004, 1991) y Perú (Ley 27669, 2002). Estos, han dispuesto una jornada laboral (tiempo al que se compromete un trabajador a laborar al servicio de un empleador, dentro de una relación laboral, puede ser diurna o nocturna y se estructura por turnos de 8, 12, 16 o 24 horas diarias con sus respectivos descansos) semanal de entre 30 y 36 horas, cuando en Colombia es de 48 y en algunos casos 56 horas. Otro ejemplo es Chile, que otorga periodos de vacaciones, permisos y licencias por casos especiales como violencia de género, matrimonio, enfermedad o calamidad (Ley 20, 2012).

A pesar de las denuncias de las agremiaciones del sector (ASNEC, 2014), los estudios que han mostrado la altísima relevancia práctica y social de estos profesionales (Torres, 2004) y su escasez en el país, -en donde en vez de 5.2 profesionales por cada 1.000 habitantes (Ruiz, et al., 2009) solo se tiene 0.6; a diferencia de Perú, que registra una tasa de 1.3 , Brasil 6.4 y Estados Unidos 9.8. (OMS, 2015) - poco se observan condiciones de trabajo que favorezcan el pleno desarrollo de la profesión y sus profesionales.

Como propuestas de solución organismos internacionales (Organización Internacional del Trabajo, 1977) han recomendado adoptar medidas para que el personal de enfermería se forme, goce de las mismas condiciones que el resto de los trabajadores y se garantice su retención; a nivel teórico se ha sugerido que las empresas asuman mayor responsabilidad en la gestión de las competencias de sus trabajadores (Vega-Monsalve, 2016) y a nivel nacional se discute el proyecto de Ley 176 de 2014 que aunque adoptaría el convenio 149 de la OIT-luego de 37 años-, seguiría en deuda con las jornadas laborales más bajas.
No incluir ninguna mejora en este tema vuelve profundamente prioritario la realización de investigaciones como ésta que pretende comprender la influencia de dichas condiciones de trabajo en la salud del personal de enfermería; al conocer las condiciones actuales pueden proponerse estrategias de intervención y a la fecha ninguna investigación que recoja este objetivo se ha realizado en el territorio Antioqueño, lo que imprime novedad y relevancia al estudio y más si se tiene en cuenta que el país atraviesa por una acelerada tendencia de flexibilización laboral donde las condiciones de empleo son desmejoradas (Vega-Monsalve et al., (2014).

\section{MÉTODOS}

Este estudio aprovechó las bondades de los dos tipos de investigación (cualitativa y cuantitativa). Luego de los cálculos estadísticos y análisis de los datos numéricos, se procedió a darle profundidad a los resultados, por medio de la comprensión de la incidencia de las condiciones de trabajo en la salud y vida del personal de enfermería, de allí la decisión de triangular métodos desde los instrumentos utilizados.

Enfoque cuantitativo. Estudio transversal sobre una muestra de 365 enfermeros Colombianos de 39 municipios de Antioquia. Al no disponer del listado del personal de enfermería que labora en la región, fue necesario realizar un muestreo no probabilístico asumiendo como universo una población infinita, estableciendo un 5\% de error y $95 \%$ de confianza. Los criterios de inclusión fueron: Hombres (n42) y mujeres (n323) entre los 18 y 58 años de edad, con más de un año de experiencia en la profesión, principalmente con estudios técnicos $(76 \%)$, de los estratos socioeconómicos $2(31.7 \%)$ y $3(48.2 \%)$, solteros $(56.7 \%)$ y con 
conyugue (36.7\%), que laboraban en instituciones de salud públicas y privadas. Los municipios fueron seleccionados a conveniencia, especialmente por la cercanía con la sede del grupo investigador (Medellín) y la facilidad en el contacto para la gestión de los consentimientos informados.

Procedimiento. Se construyó un directorio propio, los datos de contacto de los enfermeros fueron recolectados mediante la técnicas del voz a voz, se solicitó permiso a instituciones de salud para que permitieran el acercamiento con sus empleados, se promocionó el proyecto a través de Facebook y asociaciones de profesionales o egresados, se motivó a enfermeros de algunos municipios para que sirvieran como auxiliares en la recolección de los datos- estos previamente fueron entrenados - y aplicaran el cuestionario a sus colegas. Con estas estrategias se logró consolidar un directorio con la información de 1.057 profesionales quienes suministraron su consentimiento.

Enfoque cualitativo. La unidad de análisis correspondió a 21 enfermeros que previamente habían diligenciado el cuestionario, se realizaron entrevistas presenciales, se buscó la comprensión y entendimiento de los sentidos de los discursos de los sujetos.

Para asegurar el rigor y la credibilidad del estudio se cumplieron varios de los ítems contenidos en la lista de comprobación para la evaluación crítica de investigaciones cualitativas (COREQ) descritas por Tong et al., (2007). Muy especialmente se siguieron las recomendaciones del análisis y procesamiento de los datos.

Consideraciones éticas. Este estudio cumplió con lo estipulado en la Resolución 8430 de
1993 de Colombia. Está acorde con los planteamientos de Emanuel (1999) que aunque se refieren a estudios clínicos, presenta recomendaciones que en opinión del autor, todas las investigaciones deberían cumplir. De ahí el énfasis en el desarrollo de un estudio con valor social y validez científica, conveniente y transparente con la muestra. Se contó con aval del comité ético de la institución y se solicitó consentimiento firmado a los participantes.

Recolección de datos. Entre los meses de mayo y junio de 2015 se envió a la muestra el cuestionario que contenía 91 preguntas y que tomaba entre 15 y 25 minutos diligenciarlo. Se recibieron 248 encuestas, es decir, un porcentaje de respuesta del 23.4\%. Para ampliar la muestra se programaron viajes a municipios cercanos a Medellín, en donde se aplicaron 109 cuestionarios en los puestos de trabajo y de forma manual. Posteriormente fueron realizadas las entrevistas.

Instrumentos. Se aplicaron 365 cuestionarios -con opción de múltiple respuesta y auto aplicables- a través de un formulario de Google y 21 entrevistas de entre 50 y 70 minutos, grabadas con consentimiento de los participantes y luego transcritas fielmente.

Para garantizar la consistencia y fiabilidad del estudio, los instrumentos fueron construidos a partir de la comprensión teórica de las categorías y sometidos a juicio de expertos -revisaron que el contenido de las preguntas diera cuenta de las categorías estudiadas y el lenguaje y estilo de redacción fuera comprensible- y prueba piloto. Una de las variables de las condiciones de salud es el bienestar psicológico, por ello se aplicó la escala de bienestar psicológico de Ryff de 29 ítems definidos por Díaz et al., (2006). Los instrumentos in- 
dagaron por las variables de: Condiciones de salud19 y condiciones de trabajo. (Da Silva, 2006; OIT, 2014). (Ver tabla 1)

Tabla 1. Dimensión y categorías abordadas en el trabajo de campo y análisis de este estudio hipótesis definidas por el investigador. Para el procesamiento de los datos se utilizó SPSS versión 22.

\section{RESULTADOS}

Las condiciones de trabajo especialmente

\begin{tabular}{|c|c|}
\hline Dimensión & Categorías indagadas \\
\hline $\begin{array}{l}\text { Percepción de las } \\
\text { condiciones de salud }\end{array}$ & $\begin{array}{l}\text { Historial médico (enfermedades, incapacidades, intervenciones } \\
\text { quirúrgicas, hospitalizaciones, afecciones médicas, accidentes } \\
\text { de trabajo) hábitos de vida saludable y escala de bienestar } \\
\text { psicológico de Ryff que midió: Auto aceptación, relaciones } \\
\text { positivas, autonomía, dominio del entorno, crecimiento personal } \\
\text { y propósito en la vida. } \\
\text { Cargo, tipo de contrato, demandas y controles del empleo, } \\
\text { participación en las decisiones, clima laboral, remuneración, } \\
\text { condiciones sanitarias del puesto, horarios, jornadas de trabajo, } \\
\text { factores de riesgo, satisfacción laboral y tiempo de } \\
\text { desplazamiento entre el lugar de residencia y el trabajo. }\end{array}$ \\
\hline Fuente: Elaboración propia & \\
\hline
\end{tabular}

Para ver el instrumento utilizado en la recolección de datos por favor diríjase al siguiente enlace: http://goo.gl/forms/xJVzlij4X3

Análisis de datos. Los datos cualitativos fueron fielmente registrados y luego transcritos. Más tarde procesados con el software Atlas Ti. Se utilizó la técnica de análisis del discurso buscando comprender los sentidos expresados por los participantes, identificación y clasificación estructural de dimensiones estratégicas y búsqueda de patrón de comportamiento común.

Para el análisis cuantitativo se realizaron cálculos estadísticos descriptivos y se aplicó el test estadístico de chi cuadrado el cual se utiliza para buscar posibles relaciones entre dos o más grupos de estudio con respecto a una variable categórica, contrastando dos referidas a las jornadas laborales tienen una alta influencia en las condiciones de salud del personal de enfermería de Colombia del territorio Antioqueño. El 60\% (n218) labora 12 horas diarias y el 15.3\% (n56) entre 58 y 96 horas a la semana. También se les exige estar en su puesto de trabajo antes del turno y después de él para los respectivos empalmes, tiempo que no es reconocido como laboral. El 83.8\% (n306) cuenta con contrato laboral. Un 63.5\% a término indefinido.

Existe relación entre el nivel de estudios y el cargo desempeñado, no se registraron casos de personal sub o sobre calificado. En un 75\% el cargo que desempeñan es auxiliar de enfermería (n273). En cuanto a las condiciones de salud durante el último año el 44.7\% (n163) de la muestra estuvo incapacitado,-golpes o caídas al realizar esfuerzo físico con pacientes, 
migrañas y estrés- el 24.9\% (n91) sufrió de alguna enfermedad, el 11.8\% (n43) fue sometido a intervención quirúrgica y el 7.1\% (n26) estuvo hospitalizado.

Del total de la muestra el $10.7 \%$ sufrió algún tipo de accidente laboral y el 34.5\% ha sentido que su salud se ha deteriorado a raíz del trabajo que realiza. Las enfermedades que indican están directamente relacionadas con los factores de riesgos de su cargo: Escoliosis, Fibromialgia, Síndrome metabólico, Trastorno del sueño, Migraña y Estrés. Refieren la presencia de factores de riesgo psicosocial (Ver tabla 2)

Tabla 2. Condiciones de trabajo relacionadas con la salud del personal de enfermería del departamento de Antioquia, Colombia. En una escala de 0 a 10.

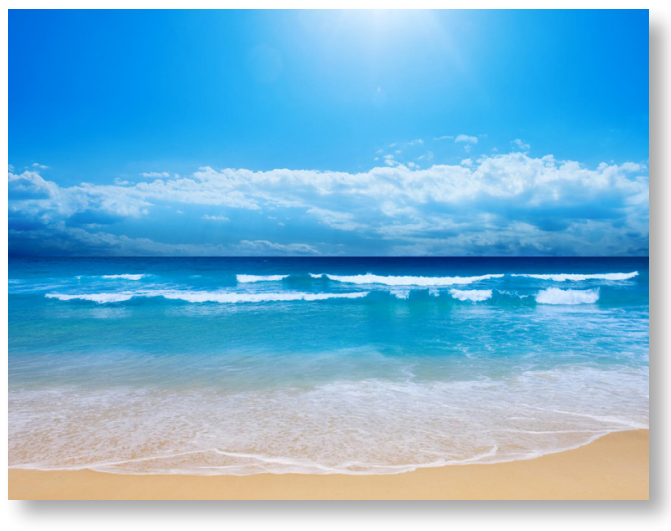

cala de 0 a 10 , señalan un 9.3, mientras que los profesionales 8.7. Por el contrario, éstos señalan que la mayor demanda es el nivel de formación especializada (9.2 vs 8.9). Respecto a la salud mental reflejada en el estado de bienestar psicológico las dimensiones de auto aceptación, relaciones positivas y propósito en la vida presentan resultados muy alejados de la media esperada, mientras que autonomía y

\begin{tabular}{|l|c|}
\hline \multicolumn{1}{|c|}{ Característica } & $\begin{array}{c}\text { Media del total } \\
\text { de la muestra }\end{array}$ \\
\hline Mi trabajo me exige estar concentrada y con atención continua & 9,2 \\
Mi trabajo me exige realizar los mismos movimientos una y otra vez & 7,9 \\
En mi trabajo tengo que aprender cosas nuevas & 8,8 \\
En mi trabajo realizo muchas tareas repetidas & 8,6 \\
En mi trabajo requiero ser creativo y resolver problemas & 8,7 \\
En mi trabajo me permiten tomar muchas decisiones por sí mismo & $* 6,5$ \\
Mi trabajo requiere un alto nivel de capacitación & 9,0 \\
Tengo muy poca libertad para decidir cómo realizar mi trabajo & $* 5,7$ \\
En mi trabajo puedo hacer muchas tareas distintas & 7,3 \\
Mi trabajo requiere trabajar muy rápido & 8,2 \\
Tengo tiempo suficiente para realizar mi trabajo & 6,7 \\
No recibo órdenes contradictorias dadas por otros & $* 5,4$ \\
Las relaciones con mis compañeros de trabajo son cordiales & 8,1 \\
Existe un muy buen clima laboral en el lugar de trabajo & 7,3 \\
*de alta relevancia para el estudio & \\
Fuente: Elaboración propia & \\
\hline
\end{tabular}

La mayor demanda de concentración y atención continua es referida por el personal con estudios técnico y tecnológico en una es- crecimiento personal son mucho más cercanos a lo esperado. (Ver tabla 3) 
Tabla 3. Resultados dimensiones del Bienestar Psicológico del personal de enfermería del departamento de Antioquia, Colombia. inesperada y renuncias. Los participantes perciben afectación de su salud sobre todo cuando: las jornadas de trabajo son muy continuas, no hay equilibrio entre la carga laboral y los descansos asignados y se considere el pos-turno como un descanso.

Los participantes indicaron que las extensas jornadas de trabajo y el hecho de que tengan que estar disponibles a cualquier hora los 365 días del año les impide hacer vida social y mantener relaciones sociales estables y duraderas,

Las sub dimensiones de autonomía, asociada a la resistencia a la presión social y a la autorregulación del comportamiento y el crecimiento personal que se manifiesta por el interés de crecer y desarrollarse como persona y profesional, presentaron resultados saludables.

Al observar la relación de jornadas de trabajo y salud, los análisis estadísticos arrojan una relación significativa. Con una confianza del 95\% y un valor $\mathrm{p}<0,05$ se halló que las horas laborales diarias se relacionan con la variable "siente que su salud de ha disminuido a raíz de su trabajo". El personal que trabajó menos de 12 horas al día registró en el último año un $1.4 \%$ de accidentes laborales, mientras que quienes trabajaron 12 horas o más al día elevaron ese porcentaje a $2.7 \%$ y $6.3 \%$ respectivamente. Las jornadas laborales iguales o superiores a doce horas influyen más en otros factores de la salud del personal que labora menos de 12 horas. (Ver tabla 4)

Los análisis cualitativos arrojaron que el número de horas de trabajo se ve afectado por festivos, actividades extra laborales como: capacitaciones, cubrir incapacidades de otros compañeros o permisos concedidos de forma existe una alta referencia a no poder cuidar de sus hijos y acompañarlos en momentos especiales de su crecimiento como actividades culturales o deportivas.

\section{DISCUSIÓN}

Se quiso comprender la influencia de las condiciones de trabajo especialmente referidas a las jornadas laborales en la salud del personal de enfermería de Colombia del territorio Antioqueño y se descubrió una alta incidencia. Preocupa que se supere el límite máximo de la jornada laboral semanal permitida por el gobierno, porque las afectaciones de salud para la muestra serían mayores a las referidas por la literatura (Manenschijn, 2011; Lana, 2014) y más cuando la jornada laboral en el país es superior a la de otros países de la región (Ley 24004, 1991; Ley 27669, 2002).

Las condiciones de salud encontradas concuerdan con los hallazgos de otras investigaciones, como las de Velandia, (2006); Manenschijn, (2011) y Ramírez, (2013) en cuanto a que a mayor número de horas laboradas mayor es el riesgo de padecer accidentes de trabajo. En otro sentido, a diferencia de las investigaciones que indican políticas de 


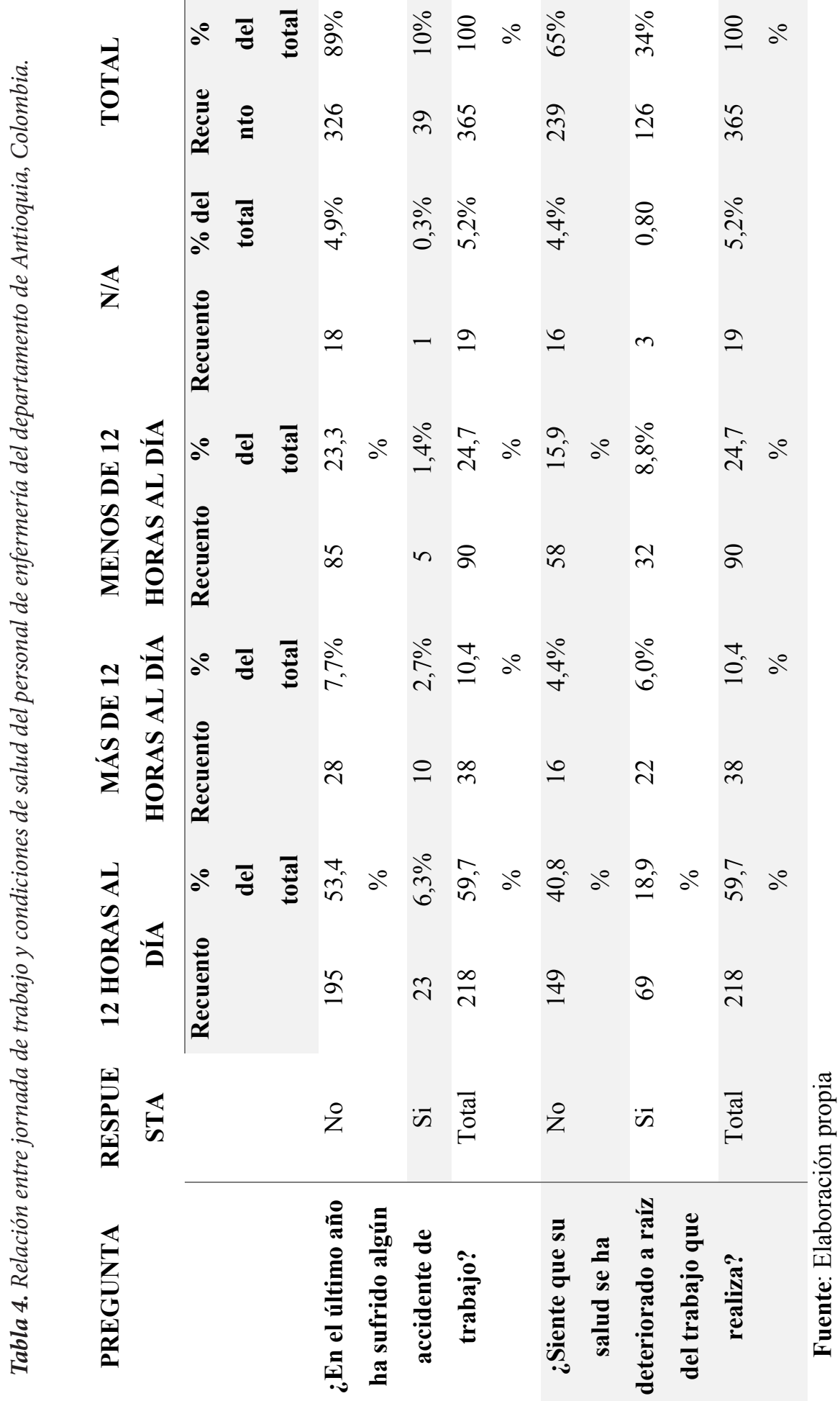


flexibilidad numérica (ASNEC, 2014; Vega-Monsalve, et al., 2014) el alto porcentaje de la muestra que cuenta con contratos laborales a término indefinido podría estar indicando una estrategia de retención por parte de las instituciones de salud, para enfrentar la escasez de este personal en el país (Ruiz et al., 2009; OMS, 2015) también a los instrumentos utilizados en este estudio y el contexto municipal en el que fueron aplicados.

Llama la atención los resultados negativos frente a factores de riesgo psicosocial, (Ver tabla 2) que son percibidos como mayores a los factores físicos y biológicos propios de este tipo de empleos. Se esperaría que el personal de enfermería esté más preocupado por un accidente biológico que por la libertad para decidir sobre cómo realizar sus funciones. Igual los resultados de bienestar psicológico que pueden interpretarse como que el promedio del personal encuestado poco se siente bien consigo mismo y mantienen relaciones sociales poco estables. Esto último, agravado según sus respuestas por la incertidumbre e intensidad de las jornadas de trabajo que les deja poco tiempo para la vida social y comunitaria.

Este estudio genera importantes contribuciones. En el sentido académico abre nuevos interrogantes que se recomienda indagar. Por ejemplo: ¿Cuáles son las razones para que las instituciones de salud programen jornadas de más de 10 horas diarias?, ¿qué inspecciones realizan las instituciones de control para que esto no suceda? y ¿cómo afectan las actuales condiciones de salud del personal de enfermería la calidad en la prestación del servicio a los usuarios? Preguntas que no fueron abordadas aquí por no ser el objeto del estudio pero que darían una comprensión integral de este fenómeno. A nivel práctico se expone las repercusiones para la salud de las condiciones precarias de trabajo.

\section{CONCLUSIONES}

A partir de esta investigación se concluye que las condiciones de trabajo desmejoran las condiciones de salud del personal de enfermería consultado, a nivel teórico se expone los efectos negativos que ocasionan las condiciones de trabajo precarias. A nivel metodológico se logró resolver el objetivo del estudio, los instrumentos utilizados dieron cuenta de las variables y categorías abordadas. Se sugiere en nuevos estudios ampliar el tamaño y ubicación de la muestra, consultando por ejemplo a los directivos de las instituciones de salud, así como a los responsables de organizar las jornadas de trabajo.

La investigación cuenta con una serie de limitaciones referidas especialmente a la ubicación de la muestra, pues no se tuvo en cuenta a todos los municipios del departamento o instituciones de salud ubicadas en zonas apartadas o rurales que pueden presentar condiciones de salud y trabajo distintas. Lo que sería interesante abordar en otro estudio.

A nivel práctico, las instituciones de salud consultadas presentan el reto de analizar las condiciones de trabajo de su personal y proponer acciones de mejora.

\section{REFERENCIAS:}

- Asociación Nacional de Enfermeras de Colombia. (2014). La enfermería una fuerza para el cambio. Recuperado de http://www.anec.org.co/revista77/revista\%20 77.pdf.

- Costa, G. (2010). Shift work and health: Current problem and preventive actions. Safety and Health at Work, (1), 112-123. Recuperado de https://www.ncbi.nlm.nih.gov/ pmc/articles/PMC3430894/

- Da Silva M. (2006). Nuevas perspectivas de la calidad de vida laboral y sus relaciones con la eficacia organizacional. Barcelona: Universidad de Barcelona.

- Díaz, D., Rodríguez-Carvajal, R., Blanco, A., MorenoJiménez, B., Gallardo, I., Valle, C. y Dierendonck, D. 
(2006). Adaptación española de las escalas de bienestar psicológico de Ryff. Psicothema, 18(3), 572-577. Recuperado de http://www.psicothema.com/psicothema. asp?id=3255

- Emanuel E. (1999). ¿Qué Hace Que la Investigación Clínica Sea Ética? Siete Requisitos Básicos. Investigación en Sujetos Humanos: Experiencia Internacional. Ed. Por A. Pellegrini Filho y R. Macklin. Programa Regional de Bioética. División de Salud y Desarrollo Humano. Organización Panamericana de la Salud/Organización Mundial de la Salud. Serie Publicaciones.

- Ley Nº. 24004. Diario Oficial de la República de Argentina, Buenos Aires, 28 de octubre de 1991.

- Ley Nº. 27669. Diario Oficial de la República de Perú, Lima, 31 de enero de 2002.

- Manenschijn, L., Van KruysBergen, R.G., de Jong, F.H., Koper, J.W. y Van Rossum, E.F. (2011). Shift work at young age is associated with elevated long-term cortisol levels and body mass index. J Clin Endocrinol Metab, 96 (11) doi: 10.1210/jc.2011-1551

- Organización Internacional del Trabajo. (1977). Convenio 149 sobre el empleo y condiciones de trabajo y de vida del personal de enfermería. Recuperado de http://www. ilo.org/dyn/normlex/es/f?p=NORMLEXPUB:12100:0:: NO::P12100_INSTRUMENT_ID:312294

- Organización Mundial de la Salud. (2015). Atlas mundial de la fuerza laboral sanitaria. Recuperado de: http://gamapserver.who.int/mapLibrary/default.aspx

- Ramírez-Elizondo, N., Paravic-Klijn, T. y ValenzuelaSuazo, S. (2013). Riesgo de los turnos nocturnos en la salud integral del profesional de enfermería. Index Enferm, (22)3: 152-155. Recuperado de http://dx.doi. org/10.4321/S1132-12962013000200008

- Real Decreto-Ley Nº. 20. Diario Oficial de la República de Chile, Santiago, Chile, 14 de julio de 2012

- Ruiz, F., Matallana, M.A., Amaya, J.L., Vásquez. M.E., Parada, L.A., y Piña MR. (2009). Recursos Humanos de la Salud en Colombia. Balance, competencias y prospectiva. Bogotá: Javegraf.

- Romero-Ballén, M. y Mesa-Melgarejo L. (2010) Profesionales de enfermería y cuidado en las condiciones laborales actuales. Rev enf. 12 (2): 55-92.

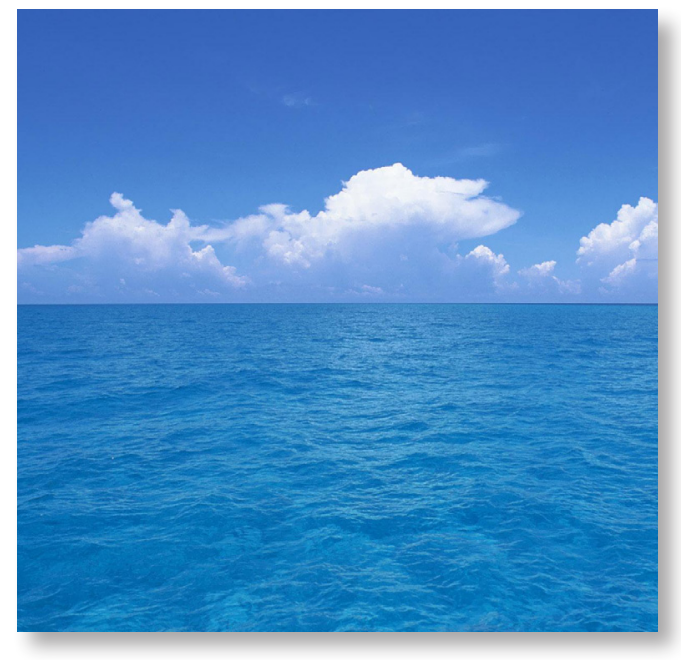

- Tong, A., Sainsbury, P., y Craig, J. (2007). Consolidated criteria for reporting qualitative research (COREQ): A 32-item checklist for interviews and focus groups. International Journal for Quality in Health Care, 19(6), 349-357.

- Torres, J. (2004). Reflexiones sobre funciones del personal de enfermería. Rev Cubana Salud Pública, 30(4),0-0. Recuperado de http://scielo.sld.cu/scielo.php?script=sci_ arttext\&pid=S0864-34662004000400009\&lng=es\&tlng $=$ es.

- Vega-Monsalve, N.D.C., Pinzón-Salgado, A.M., Álvarez, C., y Serna-Gómez, H.M. (2014). Papel de gestión humana en procesos de flexibilidad numérica en organizaciones colombianas. AD-minister: 139-164. Recuperado de http://www. scielo.org.co/scielo.php?script $=$ sci $\operatorname{arttext} \&$ pid $=$ S1692-

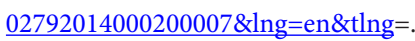

- Vega Monsalve, N.D.C. (2016.). Neocompetencias, nuevo enfoque de competencias laborales en Salud Ocupacional. Educación Médica Superior, 30(3), 627-638. Recuperado de http://scielo. sld.cu/scielo.php?script=sci_arttext $\&$ pid $=$ S086421412016000300016\&lng=es\&nrm=iso\&tlng=es

- Velandia-Mora, A.L. (2011). La enfermería en Colombia 1990-2010. Ejercicio profesional y situación legal. Imagen y Desarrollo,13(1), 65-78. Recuperado de http:// revistas.javeriana.edu.co/index.php/imagenydesarrollo/ article/viewFile/1634/1055 